CLINICAL PRACTICE

\title{
Diabetic retinopathy - the Ophthalmology Society of Southern Africa screening programme
}

\author{
S Cook \\ Steve Cook is an ophthalmologist with an interest in developing screening methodologies that enable early detection of treatable disease. He works in \\ private practice and has sessional appointments at Frere and Cecilia Makiwane Hospitals, East London, and a joint appointment at Walter Sisulu \\ University.
}

This article is presented on behalf of the Ophthalmology Society of South Africa Diabetic Retinopathy Working Group.

Corresponding author: S Cook(dr@eyecentre.co.za)

Screening for diabetic retinopathy (DR) not only allows for detection of microvascular complications, but for detection of other comorbidities. Recent advances in digital camera technology have improved screening for DR and many countries have established systems that screen all diabetics for DR annually. However, South Africa has lagged behind due to pressures at the primary care level, with the result that many diabetics are not screened. In response, the Ophthalmology Society of Southern Africa has developed a low-cost 'scorecard' system for a national DR screening programme.

S Afr Med J 2013;103(7):449-451. DOI:10.7196/SAMJ.7136

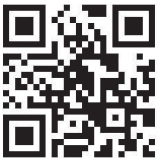

Diabetes causes microvascular complications in the retina, which commonly manifest as diabetic retinopathy (DR). DR is the leading cause of blindness in the current worldwide diabetes epidemic. Therefore, screening for DR is worthwhile and provides an opportunity to detect other comorbidities, such as glaucoma and age-related macular degeneration. Examination of the posterior pole of the eye (optic disc, retinal arcades and macula) detects a high proportion of patients with referable or treatable pathology. In addition, vital information is obtained regarding the state of the microvascular system in general.

Direct fundoscopy has traditionally been the method of examining the fundus at the primary healthcare level. The pressure of too many patients and de-skilling of healthcare workers have resulted in fewer patients being screened for DR at the primary level. However, recent advances in digital camera technology have made it possible to acquire high-resolution images of the fundus to enable screening for DR. Many countries have established DR screening systems that screen all diabetics for DR annually. South Africa (SA) has lagged behind in this regard, with the result that many diabetics are not screened. There is also a high default rate among those patients who are screened, reflecting a poor understanding of the necessity of the screening and ignorance of the import of the DR level or 'score' at any point in time.

In response, the Ophthalmology Society of Southern Africa (OSSA) has developed a system for a national DR screening programme. It is anticipated that this will incorporate screening at all levels from the clinic to the specialist.

Diabetic retinopathy screening may be described as structured or opportunistic. Examples of structured programmes would be the programmes established in the UK, funded by governments or managed health organisations. Such programmes work best in situations where the state is the major healthcare provider and there is not necessarily a strong link between the healthcare provider and the patient. Germany provides an example of a country where DR screening tends to be opportunistic and where patients access the services of their chosen healthcare provider in more individualised 'doctor-patient' relationships. Opportunistic systems tend to be funded by the individual.
In SA, the public sector tends to follow the structured model and the private sector tends toward the opportunistic model. There are recommendations for screening in place, ${ }^{[1]}$ but there is no central register, recall system or even a common grading system. A local exception to this is the Centre for Diabetes and Endocrinology (CDE) programme for DR screening, which has demonstrated the benefit of a structured approach to DR screening in SA conditions. It has also highlighted some of the modelling pitfalls where patients may be inadvertently referred away from their established eye caregivers (opportunistic model) for screening to other practitioners (structured model).

There is a general lack of education among patients regarding the need for screening. Private patients are often not referred for screening. This may be simply because the primary caregiver does not think of it and patients are not aware enough to request screening. The public sector is generally swamped with established pathology, to the extent that further screening is discouraged as it simply makes the backlog greater. Many diabetics in SA are therefore not screened for DR and often present to the ophthalmologist for the first time with vision loss due to advanced eye disease. Both our opportunistic and structured systems have failed these patients, many of whom face blindness. There is an urgent need to turn this situation around and to enable patients to know the level of severity of their DR and provide them with the necessary information to modify their risk factors and change their health-seeking behaviour.

Laser photocoagulation has been the mainstay of treatment for DR. It continues to be the key intervention, with new technologies allowing faster and less invasive therapies. Laser is, however inherently destructive. The most dramatic recent advance in the management of DR has been the use of intravitreal injections of anti-vascular endothelial growth factor (anti-VEGF) agents. These agents have demonstrated an ability to stabilise and control most of the sight-threatening DR-related disease. They are effective against the proliferative and maculopathic elements of the disease. This has meant that there is a significant increase in the ability of the ophthalmologist to treat DR and prevent blindness.

The OSSA proposes a low-cost system that makes use of the current resources available to improve access to screening, and is adaptable to the local conditions within the structured or opportunistic models. 
A public awareness campaign needs to be undertaken to motivate diabetic patients to seek screening.

All willing and able screeners need to be recruited and trained so as to be able to offer access to screening. Direct fundoscopy, fundus photography, slit-lamp and indirect ophthalmoscopy all play a role as individual modalities, and in combination, in the examination for diabetic eye disease. The OSSA is aware that these are often not available or not used for screening by the primary caregiver. We wish to promote our screening programme as an educational exercise for patients and caregivers. Where patients are not able to access screening from their primary caregiver we would encourage referral for screening to an appropriate centre. This may require that the patient be referred to the private sector from the public sector or vice versa. It may mean that they may be referred from a GP to another GP, an optometrist or an ophthalmologist. We propose an accreditation system to provide quality assurance $(\mathrm{QA})$.

\section{The OSSA DR screening system}

Our goal is to develop a patient-driven diabetic screening programme that identifies and informs health-seeking behaviour of high-risk patients.

The realities of the healthcare scenario in SA at present mean that there will not be a systematic screening system in the immediate future. The key principle of the OSSA system is the clear transfer of responsibility for health-keeping to the patient. We want to make it possible for all levels of practitioner to participate. This would include nursing sisters, optometrists, GPs, specialist physicians, endocrinologists and ophthalmologists.

There are four key elements of the screening system, which make use of the best elements of the opportunistic system and some of the benefits of the structured system:
1. A common DR screening grading system. The Scottish DR grading system has been adopted to develop a simple featurebased grading system (Fig. 1). It is hierarchical with increasing severity from microaneurysms $<$ blot haemorrhages $<$ intraretinal microvascular anomalies < venous beading $<$ new vessels. It is based on the examination of the posterior pole of the eye (the optic disc, temporal arcades and macular area). This is highly suited to examination with a direct ophthalmoscope and has a wellestablished evidence base for single field digital fundus screening. 2. An internet-based DR database and patient-tracking system. This is a closed user, group database that functions as a medical record for the participant screeners' patients and a common database for the whole screening programme. Screeners can access their own patients via their file numbers. In a situation where a patient migrates between systems or practices their database records can be accessed by means of their individual database number. Confidentiality is maintained by the common database being 'denatured'. Patient consent to be loaded onto the database is requested by the screener.

3. A patient-held record (PHR). It is common for patients to leave a consultation without knowing what was found and its significance. This means that they cannot relay vital information to their families and primary caregivers. The communication between the point of screening and the primary caregiver is often inadequate. When the patient meets the primary caregiver, there is often more confusion as there are a number of possible classifications of DR and the primary caregiver may not be familiar with these. The PHR attempts to bridge these gaps using the popular concept of a sports 'score'. The PHR 'scorecard' is marked with the observed grade, the follow-up date and any other disease present. The patient takes the record as a reminder of the follow-up and as a communication tool.

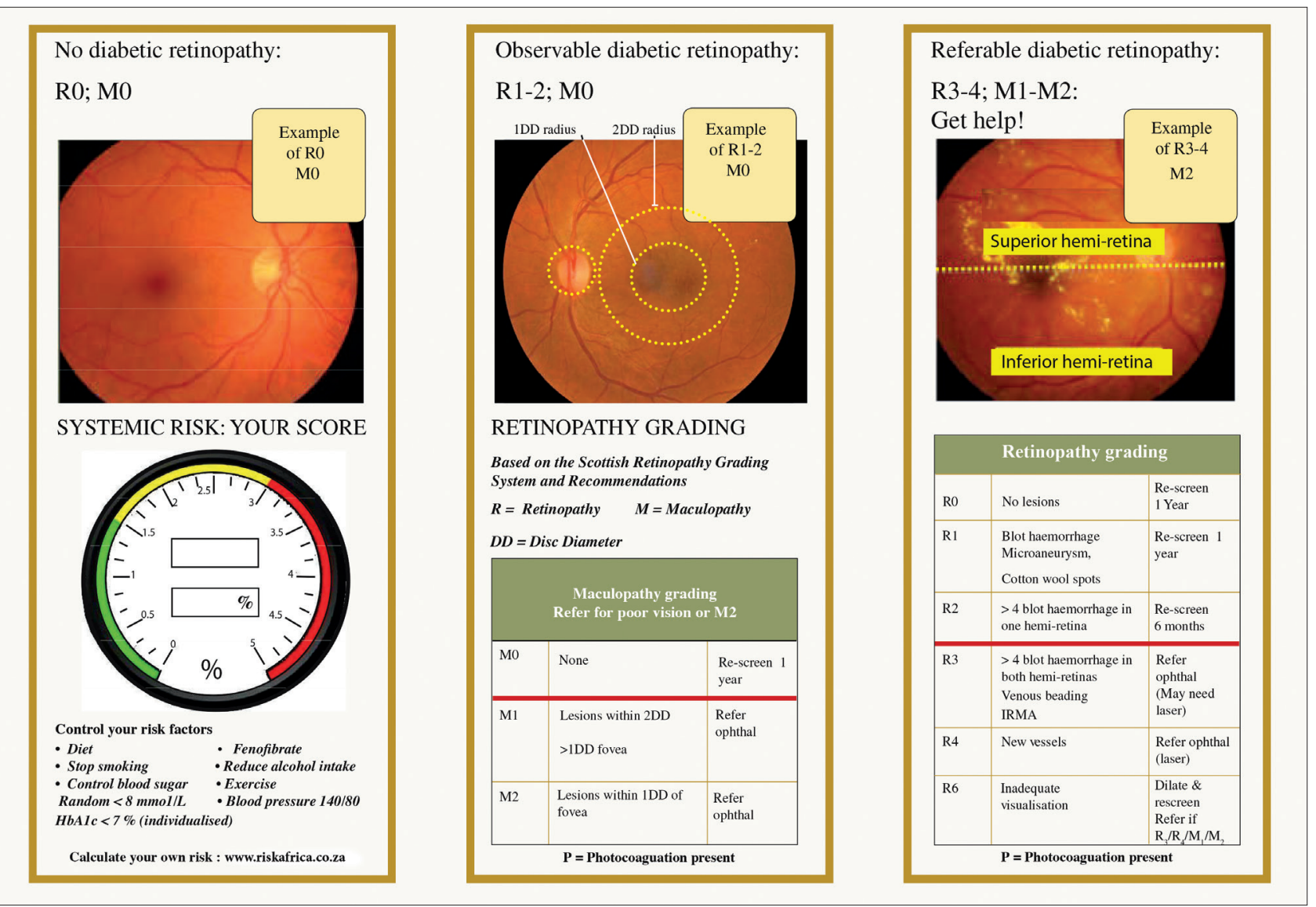

Fig. 1. Examples of the OSSA DR screening system taken from the PHR. 
The card is marked with their database number as a cross reference should they migrate between practitioners.

Control of systemic risk remains the most important factor in preventing diabetic eye disease. Systemic risk for progression to sight-threatening eye disease is incorporated into the PHR. A risk calculator has been developed for SA conditions in collaboration with Prof. Einar Steffansson, who developed a risk calculator based on the outcomes observed in a large Danish cohort. ${ }^{[2]}$ Systemic risk is calculated and the outcome drawn onto a 'rev counter' that is placed next to the 'no retinopathy' photographs (Fig. 1). This helps dispel the possible misconception that no retinopathy means 'no need to control the systemic risk factors'.

4. A QA system. A collaboration has been established with the University of Aberdeen to make use of their digital QA system. This internet-based system has been in use in Scotland to evaluate graders. The system plots the sensitivity and specificity of graders on a receiver operating characteristic (ROC) curve. This allows graders to see where they are with respect to their peers. The system has been found to be highly motivational and educational. The graders generally show improvement in QA outcomes over time.

\section{The Diabetic Retinopathy Register}

The central point of communication and resource is the freely accessible online database to be found at www.diabeticregister. co.za (contact S Cook to receive a link to enrol on the site). The site contains a download function for resources such as the PHR and online training. The risk calculator for calculating systemic risk of sight-threatening retinopathy is included in the online screening and is available from Risk Medical Solutions (www.riskafrica.co.za). PHRs can be ordered directly from Harry's Printers (contact deena@ harrysprinters.co.za). OSSA members have indicated their willingness to provide training for screening. All levels of healthcare practitioners are encouraged to participate.

\section{Conclusion}

The OSSA screening programme will systematically raise the level of knowledge of DR among patients and their caregivers. We anticipate that informed patients will make better choices regarding their health-seeking behaviour. Our system makes the most of the current resources by providing immediate access to a common platform and communication methodology. The involvement of all levels of healthcare practitioner should make access to screening much easier for patients. Appropriate, timely referral will afford patients the best possible opportunity to retain their sight.

1. Department of Health. Diabetic retinopathy. In: National Guideline. Prevention of Blindness in South Africa Pretoria: Department of Health, 2002:12-15. http://www.kznhealth.govza//blindness.pdf (accessed 10 June 2013), 2. Aspelund T, Thornórisdóttir O, Olafsdottir E, et al. Individual risk assessment and information technology to optimise screening frequency for diabetic retinopathy. Diabetologia 2011;54:2525-2532. [http://dx.doi.org/10.1007/s00125-011-2257-7]

Accepted 7 June 2013. 